\title{
A CONJECTURE ON WEAK COMPACTNESS
}

BY

\section{VICTOR KLEE( ${ }^{(1)}$}

There are several characterizations of weak compactness in terms of intersection properties of convex sets. The first of these was Šmulian's theorem [13] to the effect that if a closed convex set $C$ in a normed linear space is not weakly compact, then $C$ contains a decreasing sequence of nonempty closed convex sets whose intersection is empty. This result was sharpened in various ways by Dieudonné [4], Floyd and Klee [5], and Ptak [11]. (For a survey of these and related results, see Chapter III of Day [3].) However, in each of their constructions it may happen that all the sets in the sequence penetrate rather far into the set $C$; for example, if $C$ is the unit cell $\{x \in E:\|x\| \leqq 1\}$ of a normed linear space $E$, it may happen for some $m \in[0,1[$ that each set in the sequence intersects the smaller cell $m C$. On the other hand, R. C. James [7] has proved that if $C$ is the unit cell of a separable Banach space $E$ and $C$ is not weakly compact (or, equivalently, $E$ is nonreflexive), then there exists a linear functional $f \in E^{*}$ such that $\|f\|=1$ but $f x<1$ for all $x \in C$. With $K_{n}=\{x \in C: f x \geqq 1-1 / n\}$, it is clear not only that $\bigcap_{1}^{\infty} K_{i}=\varnothing$ but also that for each $m \in[0,1$ [, the set $m C$ meets only finitely many of the sets $K_{i}$.

The theorem of James lends support to the following

Conjecture. A closed convex subset $C$ of a separable Banach space $E$ is weakly compact if and only if every continuous linear functional on $E$ attains a maximum on $C$.

The conjecture reduces at once to the case of a bounded set. In the present note we supply further support by proving the following

THEOREM. Suppose $C$ is a bounded closed convex subset of a separable Banach space, and $C$ is not weakly compact. Then $C$ contains a decreasing sequence $K_{\alpha}$ of nonempty closed convex sets such that for each $x \in C$ and each $m \in[0,1$, the set $x+m(C-x)$ meets only finitely many of the sets $K_{i}$.

Note that from the above conjecture there would follow the same characterization of weak compactness for an arbitrary weakly closed subset of $E$. Further, James has announced (at a conference in Warsaw in 1960) the validity of his theorem for an arbitrary nonreflexive Banach space. Thus it may be true for any Banach space $E$ that a weakly closed subset $X$ of $E$ is weakly compact if and only if every $f \in E^{*}$ attains a maximum on $X$.

Our proof of the Theorem stated above depends on the notion of relative

Presented to the Society, November 18, 1961 ; received by the editors July 19, 1961.

(1) Research supported by the Alfred P. Sloan Foundation. 
extreme point introduced in [8] and considered also in [10]. When $Z$ is a subset of a linear space and $X \subset Z$, a point $z$ of $Z$ is said to be extreme in $Z$ relative to $X$ provided there do not exist distinct points $x \in X$ and $z^{\prime} \in Z$ such that $z \in] x, z^{\prime}$ [. The set of all such relative extreme points will be denoted by $e x_{X} Z$. Our basic tool is the existence of relative extreme points under certain circumstances, in conjunction with the following result:

1. Proposition. Suppose $X$ and $Z$ are subsets of a Hausdorff linear space, $Z$ is closed and starshaped from $X$, and every open covering of $X$ admits a subcovering of cardinality $\leqq \aleph$, where $\boldsymbol{N}$ is an infinite cardinal number. Suppose $z \in e x_{X} Z \sim X$. Then there is a set $\mathfrak{N}$ of neighborhoods of $z$ such that card $\mathfrak{N} \leqq \aleph$ and $Z \cap\left(\cap_{N \in \mathfrak{N}} N\right) \subset e x_{X} Z \sim X$.

Proof. Note that for every $x \in X$ and every positive integer $n$ there exist disjoint open neighborhoods $U(x, n)$ of $x$ and $V(x, n)$ of $z$ such that whenever $u \in U(x, n) \cap X$ and $v \in V(x, n) \cap Z$, then the ray $u+[(n+1) / n, \infty[(v-u)$ lies entirely in the complement of $Z$. For otherwise there are nets $x_{\alpha}$ in $X, z_{\alpha}$ in $Z$, and $r_{\alpha}$ in $[(n+1) / n, \infty$ [(where $\alpha$ ranges over some directed set) such that $x_{\alpha} \rightarrow x, z_{\alpha} \rightarrow z$, and always $x_{\alpha}+r_{\alpha}\left(z_{\alpha}-x_{\alpha}\right) \in Z$. Since $Z$ is starshaped from $X$, this implies that

$$
x_{\alpha}+\frac{n+1}{n}\left(z_{\alpha}-x_{\alpha}\right) \in Z
$$

whence

$$
x+\frac{n+1}{n}(z-x) \in Z
$$

for $Z$ is closed. But this contradicts the hypothesis that $z \in e x_{X} Z$.

For each $n$, the class $\{U(x, n): x \in X\}$ is an open covering of $X$, and hence there is a subset $S_{n}$ of $X$ such that card $S_{n} \leqq \aleph$ and $X \subset \bigcup_{x \in S_{n}} U(x, n)$. Let $\mathfrak{x}=\bigcup_{n=1}^{\infty}\left\{V(x, n): x \in S_{n}\right\}$. Then card $\mathfrak{N} \leqq \boldsymbol{N}_{0} \cdot \boldsymbol{N}=\boldsymbol{N}$. If $u \in X$ and $v \in Z \cap\left(\bigcap_{N \in \mathfrak{T}} N\right)$, then

$$
u+] 0, \infty\left[(v-u)=\bigcup_{n=1}^{\infty}\left(u+\left[\frac{n+1}{n}, \infty[(v-u)),\right.\right.\right.
$$

and by the choice of $\Re$ the latter set cannot intersect $Z$. Consequently $v \in \operatorname{ex}_{X} Z \sim X$ and the proof is complete.

2. Theorem. Suppose $C$ is a sequentially complete bounded convex subset of a locally convex space, $E$ is the linear extension of $C$, and $C$ is not weakly compact but does enjoy the Lindelöf property with respect to the weak topology $\sigma\left(E, E^{*}\right)$. Let $\xi$ denote the natural embedding of $E$ in the space $E^{* \prime}$ (the algebraic dual of $\left.E^{*}\right), C_{\sigma}$ the closure of $\xi C$ in the topology $\sigma\left(E^{* \prime}, E^{*}\right), E_{2}$ the linear extension of 
$C_{\sigma}$, and $\sigma_{2}$ the restriction to $E_{2}$ of $\sigma\left(E^{* \prime}, E^{*}\right)$. Then there exist a point $Y \in C_{\sigma}$ and a sequence $f_{\alpha}$ in $E^{*}$ such that with $\phi X=\sum_{1}^{\infty} 2^{-n}\left|X f_{n}-Y f_{n}\right|$ for all $X \in E_{2}$, the function $\phi$ is finite-valued, $\sigma_{2}$-continuous, and

$$
\left\{X \in C_{\sigma}: \phi X=0\right\}=\left\{X \in C_{\sigma}: X f_{\alpha}=Y f_{\alpha}\right\} \subset e x_{\xi C} C_{\sigma} \sim \xi C .
$$

If $K_{n}=\{x \in C: \phi \xi x \leqq 1 / n\}$, then $K_{\alpha}$ is a decreasing sequence of nonempty closed convex subsets of $C$ such that for each $x \in C$ and each $m \in[0,1[$, the set $x+m(C-x)$ meets only finitely many of the sets $K_{i}$.

Proof. Let $\tau$ denote the given topology for $E$ and $\sigma_{0}$ the weak topology $\sigma\left(E, E^{*}\right)$.

Since $C$ is $\sigma_{0}$-bounded, the set $\xi C$ is $\sigma\left(E^{* \prime}, E^{*}\right)$-bounded and hence $C_{\sigma}$ is $\sigma\left(E^{* \prime}, E^{*}\right)$-compact. Consequently, both $C_{\sigma}$ and the set $U=C_{\sigma}-C_{\sigma}$ must be $\sigma_{2}$-compact. Since $U$ is convex and symmetric, the gauge functional $\mu$ of $U$ is a norm for $E_{2}$ relative to which the unit cell is $U$ itself. And since $E=R(C-C)$, the function $\mu \xi$ is a norm for $E$. Let $\mu_{2}$ and $\mu_{0}$ denote the metric topologies for $E_{2}$ and $E$ which are generated by the respective norms $\mu$ and $\mu \xi$. Since $U$ is $\sigma_{2}$-bounded, $\mu_{2}$ must be finer than $\sigma_{2}$, and $\mu_{2}$-completeness of the sets $U$ and $C_{\sigma}$ is a consequence of the following fact (Grothendieck [6, p. 163]): If $\tau_{1}$ and $\tau_{2}$ are two locally convex Hausdorff linear topologies for a linear space $E$, $\tau_{1}$ is finer than $\tau_{2}$, and there exists a $\tau_{1}$-fundamental system of $\tau_{1}$-neighborhoods of 0 which are $\tau_{2}$-closed, then every $\tau_{2}$-complete subset of $E$ is $\tau_{1^{-}}$ complete.

Note also that $\sigma_{2}$-boundedness of $U$ implies $\sigma_{0}$-boundedness of the set $\xi^{-1}(U \cap \xi E)$ which is the unit cell of $E$ relative to the norm $\mu \xi$; since this set is $\sigma_{0}$-bounded it must be $\tau$-bounded (according to a theorem of Mackey [9]) and consequently $\mu_{0}$ is finer than $\tau$. This fact can be used to show that the set $\xi C$ is $\mu_{2}$-closed. For consider a sequence $X_{\alpha}$ in $\xi C$ which is $\mu_{2}$-convergent to a point $X \in C_{\sigma}$. For each $n$ there exists $x_{n} \in C$ such that $X_{n}=\xi x_{n}$, and of course $x_{\alpha}$ is a $\mu_{0}$-Cauchy sequence in $C$. But $\mu_{0}$ is finer than $\tau$, so $x_{\alpha}$ is also $\tau$-Cauchy, and since $C$ is $\tau$-sequentially-complete the sequence $x_{\alpha}$ must be $\tau$-convergent to a point $x \in C$. For each $f \in E$ we have

$$
(\xi x) f=f x=\lim f x_{\alpha}=\lim X_{\alpha} f=X f,
$$

and from this it follows that $X=\xi x \in \xi C$.

We know now that $C_{\sigma}$ is $\mu_{2}$-bounded and $\mu_{2}$-complete, and $\xi C$ is a $\mu_{2}$-closed convex subset of $C_{\sigma}$. And of course $\xi C \neq C_{\sigma}$, for we are assuming that $C$ is not $\sigma_{0}$-compact. It follows from a theorem in [8] that there exists a point

$$
Y \in e x_{\xi C} C_{\sigma} \sim \xi C .
$$

Since $C$ enjoys the Lindelof property relative to $\sigma_{0}$, the same must be true of $\xi C$ relative to $\sigma_{2}$ and thus by Proposition 1 there is a sequence $W_{\alpha}$ of $\sigma_{2}$-neighborhoods of $Y$ in $E_{2}$ such that 


$$
C_{\sigma} \cap\left(\bigcap_{1}^{\infty} W_{i}\right) \subset e x_{\xi C} C_{\sigma} \sim \xi C .
$$

Let $S^{*}=\left\{f \in E^{*}: \sup _{X \in U} X f=1\right\}$. Recalling the definition of the topology $\sigma_{2}$, we see that for each $n$ there is a finite subset $G_{n}$ of $S^{*}$ such that $W_{n}$ contains the set of all $X \in E_{2}$ for which $X g=Y g$ for all $g \in G_{n}$. Let $f_{\alpha}$ be an enumeration of the set $\cup_{n=1}^{\infty} G_{n}$ and for each $X \in E_{2}$ let

$$
\phi X=\sum_{n=1}^{\infty}\left|X f_{n}-Y f_{n}\right| / 2^{n} .
$$

Of course for each $n$ the function $X f_{n} \mid X \in E_{2}$ is $\sigma_{2}$-continuous, and since $\left|X f_{n}-Y f_{n}\right| \leqq \mu(X-Y)<\infty$ we see that $\phi$ is a $\sigma_{2}$-continuous real-valued convex function on $E_{2}$. From the choice of sequence $f_{\alpha}$ it is evident that

$$
\left\{X \in C_{\sigma}: \phi X=0\right\}=\left\{X \in C_{\sigma}: X f_{\alpha}=Y f_{\alpha}\right\} \subset e x_{\xi C} C_{\sigma} \sim \xi C .
$$

Since $\phi$ is convex and $\sigma_{2}$-continuous, inf $\phi C_{\sigma}=0$, and $\xi C$ is $\sigma_{2}$-dense in $C_{\sigma}$, it is evident that each set $K_{i}$ is convex, closed, and nonempty. Now with $x \in C$ and $m \in[0,1$ [, suppose the set $x+m(C-x)$ meets infinitely many of the sets $K_{i}$. Then inf $\phi(\xi x+m(\xi C-\xi x))=0$, and since $C_{\sigma}$ is $\sigma_{2}$-compact there exists $Z \in C_{\sigma}$ such that $\phi(x+m(Z-\xi x))=0$. Then $\xi x+m(Z-\xi x) \in e_{\xi} C_{\sigma}$, whence $\xi x=Z$ and $\phi \xi x=0$, a contradiction completing the proof.

To establish a slight generalization of the THEOREM stated earlier, it suffices to apply Theorem 2 in conjunction with the following observation:

3. Proposition. Suppose $E$ is a separable metrizable locally convex space. Then in the weak topology $\sigma\left(E, E^{*}\right)$, every subset of $E$ enjoys the Lindelof property.

Proof. We note first that if $Y$ is a separable metric space and $X$ is a topological space which is a union of countably many separable metrizable subsets, then every set of continuous maps of $X$ into $Y$ enjoys the Lindelöf property with respect to the topology of pointwise convergence. Under the assumption that $X$ and $Y$ are both separable metric, this was proved by M. E. Rudin and the author [12], but the proof required of $X$ only that the product space $] 0, \infty\left[\times X^{n} \times Y^{n}\right.$ should be separable for each $n$.

Now let $U_{\alpha}$ be a fundamental sequence of neighborhoods of 0 in $E$, and for each $i$ and $j$ let $V_{i j}=\left\{f \in E^{*}\right.$ : $\left.\sup f U_{i} \leqq j\right\}$. Then $E^{*}=U_{i, j} V_{i j}$, and since $E$ is separable every set $V_{i j}$ must be compact and metrizable under the topology $\sigma\left(E^{*}, E\right)$ (p. 66 of Bourbaki [1]). Let $\xi$ denote the natural map of $E$ into $E^{* \prime}$. Then $\xi$ is a linear homeomorphism of the space $\left(E, \sigma\left(E, E^{*}\right)\right)$ into the space $\left(E^{*}, \sigma\left(E^{*}, E\right)\right)^{*}$ under its weak topology, and every subset of the latter space is Lindelöfian by the result of the preceding paragraph. This completes the proof.

We note in closing that the THEOREM surely is valid for every Banach 
space which is Lindelöfian in its weak topology, and that there exist nonseparable spaces of this sort (see Corson [2]).

\section{REFERENCES}

1. N. Bourbaki, Espaces vectoriels topologiques, Chapters III-V, Hermann, Paris, 1955.

2. H. H. Corson, The weak topology of a Banach space, Trans. Amer. Math. Soc. 101 (1961), 1-15.

3. Mahlon M. Day, Normed linear spaces, Springer, Berlin, 1957.

4. J. Dieudonne, Sur un theoreme de Smulian, Arch. Math. 3 (1952), 436-439.

5. E. E. Floyd and V. L. Klee, A characterization of reflexivity by the lattice of closed subspaces, Proc. Amer. Math. Soc. 5 (1954), 655-661.

6. A. Grothendieck, Espaces vectoriels topologiques, São Paulo, 1954.

7. R. C. James, Reflexivity and the supremum of linear functionals, Ann. of Math (2) 66 (1957), 159-169.

8. Victor Klee, Relative extreme points, Proc. Internat. Sympos. Linear Spaces, Jerusalem, 1960, pp. 282-289, Jerusalem Academic Press, Jerusalem, 1961.

9. G. W. Mackey, On convex topological linear spaces, Trans. Amer. Math. Soc. 60 (1946), 519-537.

10. R. R. Phelps, Support cones and their generalizations, Proc. Sympos. Pure Math. vol. 7, Amer. Math. Soc., Providence, R. I. (to appear).

11. Vlastimil Ptak, Two remarks on weak compactness, Czechoslovak Math. J. 5 (80) (1955), 532-545.

12. Mary Ellen Rudin and V. L. Klee, Jr., A note on certain function spaces, Arch. Math. 7 (1956), 469-470.

13. V. L. Smulian, On the principle of inclusion in the space of type (B), Mat. Sb. (N.S.) 5(47)(1939), 317-328 (Russian with English summary).

UNIVERSITY OF WASHINGTON,

Seattle, Washington 